PAPER

\section{Shrinkage and colour in the production of micro- sized PDMS particles for microfluidic applications}

To cite this article: C F Anes et al 2018 J. Micromech. Microeng. 28075002

View the article online for updates and enhancements.
Related content

PDMS droplet formation and characterization by hydrodynamic flow focusing technique in a PDMS square microchannel

J Carneiro, E Doutel, J B L M Campos et al.

Simple and inexpensive microfluidic devices for the generation of

monodisperse multiple emulsions

Er Qiang Li, Jia Ming Zhang and Sigurdur $T$ Thoroddsen

Liquid crystals in micron-scale droplets. shells and fibers

Martin Urbanski, Catherine G Reyes, JungHyun Noh et al. 


\title{
Shrinkage and colour in the production of micro-sized PDMS particles for microfluidic applications
}

\author{
C F Anes ${ }^{1}$, D Pinho ${ }^{1,5}$, B N Muñoz-Sánchez ${ }^{2}$, E J Vega ${ }^{3}$ and R Lima ${ }^{4,5}$ \\ 1 Polytechnic Institute of Bragança, Campus de Santa Apolónia, Bragança, Portugal \\ 2 Departamento de Ingeniería Aeroespacial y Mecánica de Fluidos, Universidad de Sevilla, E-41092 \\ Sevilla, Spain \\ 3 Departamento de Ingeniería Mecánica, Energética y de los Materiales and Instituto de Computación \\ Científica Avanzada (ICCAEx), Universidad de Extremadura, E-06006 Badajoz, Spain \\ ${ }^{4}$ Minho University, MED, Guimarães, Portugal \\ 5 CEFT, FEUP, R. Dr. Roberto Frias, 4200-465 Porto, Portugal \\ E-mail: ejvega@unex.es
}

Received 21 February 2018

Accepted for publication 19 March 2018

Published 11 April 2018

\begin{abstract}
Polydimethylsiloxane (PDMS), due to its remarkable properties, is a suitable polymer for the production of microparticles with industrial and medical applications. The micro-sized PDMS liquid droplets suffer a pronounced shrinkage while curing to turn into solid particles. In this article, we report the calibration of the shrinkage phenomenon in the production of PDMS microparticles. Our results show that this shrinkage does not depend on the amount of curing agent in the PDMS precursor or on the addition of micro/nanoparticles to the mixture, but on the surface effects due to the relatively large droplet surface-to-volume ratio. Moreover, we have also investigated the addition of colour to the particles to improve their visualization/ detection. The addition of colour by using pigments enhances the visualization of the contours of the PDMS microparticles, and reveals the capability of this technique to microencapsulate micro/nanoparticles in PDMS spheres with diameters below $10 \mu \mathrm{m}$. We demonstrate that the technique used in this work is able to work with a wide range of viscosities obtaining an acceptable degree of monodispersity.
\end{abstract}

Keywords: polydimethylsiloxane (PDMS), micro-sized PDMS particles, flow focusing, shrinkage, coloured PDMS

(Some figures may appear in colour only in the online journal)

\section{Introduction}

Polydimethylsiloxane (PDMS), due to its remarkable properties, is one of the most widely used polymers in many industrial and medical applications [1-6]. In the last decade, the production of monodisperse particles of PDMS has attracted great interest among researchers because of their potential applications, especially in biomedicine [6, 7]. Microfluidics, particularly when applying the flow focusing principle, has demonstrated to be a leading platform to generate droplets and microparticles with tailored sizes and shapes [8]. A coflowing method was used to generate amphiphilic PDMS particles with hundreds of microns in size by Zhao et al [9]. Jiang et al [10] have proposed a flow-focusing technique where a PDMS precursor was dispersed into microdroplets within an aqueous continuous phase. By using this method, they were able to produce PDMS microbeads with an average dimension of 80 microns to use them as discrete oxygen sensors. Carneiro et al [11] formed and characterized PDMS droplets ( $>100$ microns) by a hydrodynamic flow-focusing technique in a PDMS square microchannel, the droplet generation was characterized and a flow regime map addressed by the capillary numbers of each phase. Nascimento et al [12] fabricated tunable elastic microcapsules (with shell of PDMS 
and sizes of hundreds microns) and studied their flow through constrictions. For many applications, it is essential to have PDMS microparticles with smaller dimensions: for example, to develop blood-analogue fluids, where PDMS microparticles will mimic red blood cells (RBCs), with dimensions below 10 microns. Recently, Pinho et al [1] and Calejo et al [2] have developed particulate blood analogues with rigid particles able to show some blood flow phenomena that happen within PDMS microchannels. However, both studies have also shown evidence that the only way to closely mimic several microscale blood flow phenomena that happen at both in vivo and in vitro environments is by using deformable microparticles. Hence, Muñoz-Sánchez et al [7] proposed a flow-focusing technique to produce such micro-sized PDMS flexible spherical particles for biomicrofluidics applications, demonstrating also the ability of the produced particulate fluid to reproduce the steady shear viscosity curve of RBCs suspended in dextran 40.

The technique developed by Muñoz-Sánchez et al [7] to produce flexible microparticles makes use of a PDMS precursor, with a proportion Part A:Part B of 6:4. This precursor is injected through a hypodermic needle to form a film/reservoir over the needle's outer surface. This film flows towards the needle tip until a liquid ligament is steadily ejected thanks to the dragging action of a coflowing viscous liquid stream of (basically) glycerol. The co-flow provides the ligament with the necessary momentum to overcome the surface tension and form a small capillary jet. The jet eventually breaks up into PDMS precursor droplets due to the growth of capillary waves, producing a micrometer emulsion. This method benefits from the remarkable stability of the fluidic structure from which the jets originate [13]. The emulsion obtained is heated up by using a common heater in order to conduct crosslinking and thermally cure the PDMS liquid droplets in the solution. Finally, the generated solid transparent microparticles can be removed from the bulk to get re-dispersed in various media depending on the application. Figure 1 shows an overview of this microparticle generation process, more details can be found in [7]. The PDMS liquid droplets and particles (before and after curing, respectively) can be visualized during this process to measure the size distribution by using microscopy and an optical imaging method.

In [7], the size distribution of the particles was analyzed, before and after curing, showing a relatively high degree of monodispersity. Surprisingly, the PDMS liquid droplets suffered a pronounced shrinkage while curing (see [7] for more details). The ability to control the size and other mechanical properties of such microparticles will enable the production of innovative and versatile delivery vehicles suitable for different biomedical applications, including blood analogue fluids $[1,2,14,15]$, magnetic hyperthermia $[4,16,17]$ and contrast agents for magnetic resonance imaging [16]. The latter applications, are based on the potential of this technique to encapsulate fluorescent and magnetic nanoparticles inside the PDMS droplets. Additionally, the simple encapsulation of different kinds of nanoparticles with high thermal performances open opportunities in a wide variety of applications such as electronics cooling [17, 18], engine cooling [17], solar thermal energy [19, 20] and cooling and heating
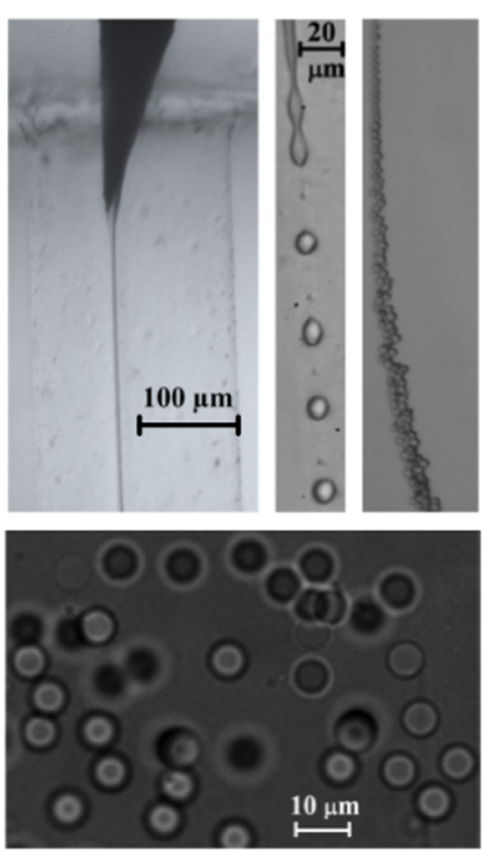

Figure 1. Images acquired in the course of the experiments along the stream of fluids: (above, from left to right) emitted jet of PDMS precursor attached to the needle tip, PDMS droplets about $10 \mu \mathrm{m}$ in diameter formed after the jet breakage, and stream of these droplets downstream the glass capillary. (Below) Inverted-microscopy image of the cured PDMS particles re-dispersed in Dextran 40 [7]. Reprinted from [7], with the permission of AIP Publishing.

in buildings [17]. Hence, this diversity of applications, makes our research interest to be devoted to a more complete understanding and characterization of this technique to produce PDMS particles.

In this work, the technique proposed by Muñoz-Sánchez et al [7] is analyzed in detail to calibrate the shrinkage phenomenon and to add colour to the particles in order to improve their later visualization/detection. In addition, our study shows the capability of the technique to work with a wide range of PDMS precursor viscosities. The paper is organized as follows. The study of the shrinkage phenomenon is presented in section 2. In section 3, we show the results of our coloured PDMS. The paper closes with the main conclusions in section 4.

\section{Shrinkage}

It is well known that PDMS shrinkage is highly variable as many parameters are involved, especially when working in a multi-user environment without tightly controlled fabrication conditions [21]. Typically, it should be characterized on a caseby-case basis, and individual calibration or alignment methods are needed for each design. Lee and Lee [22] observed PDMS shrinkage to increase with growing curing temperature, layer thickness and width, and mixing ratio of dilutant and curing agent, for theirs 2D PDMS structure. Jeong and Konishi [23] found PDMS molds/stamps shrinkage to be size-dependent, being greater for increasing dimensions. They also showed the temperature-dependence. Shrinkage is also dependent on the ambient during curing, different shrinkage velocities were found when the PDMS surface were exposed to air or not [24]. 


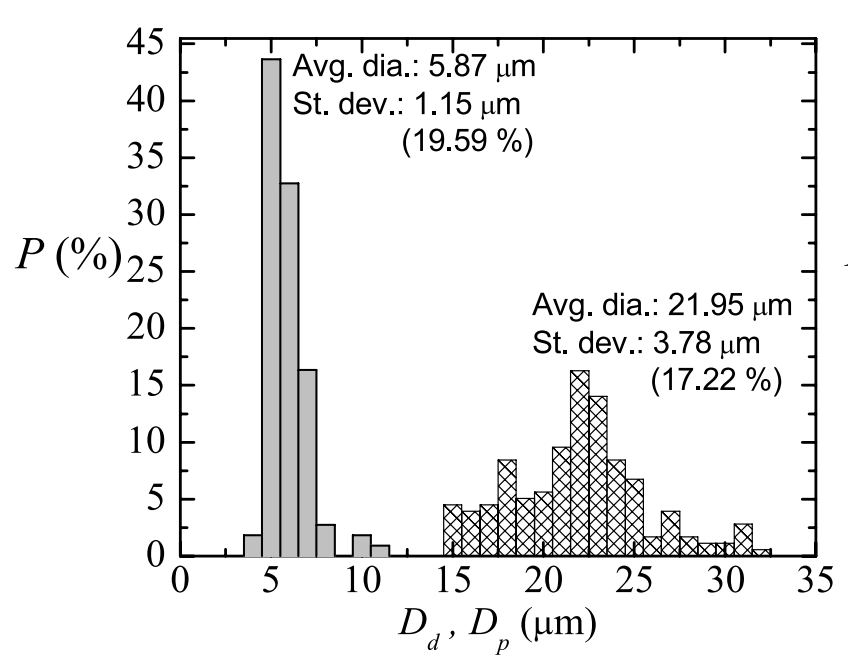

(a)

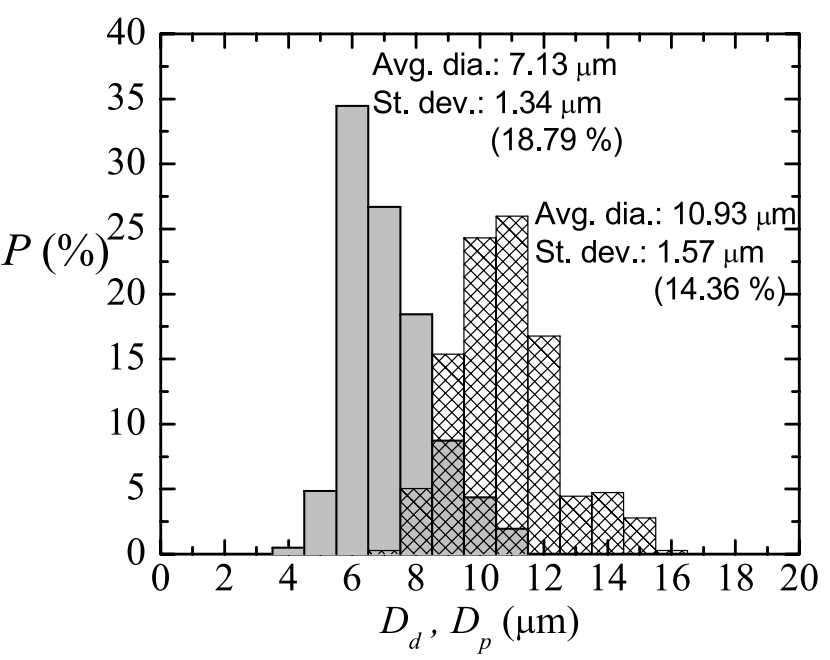

(b)

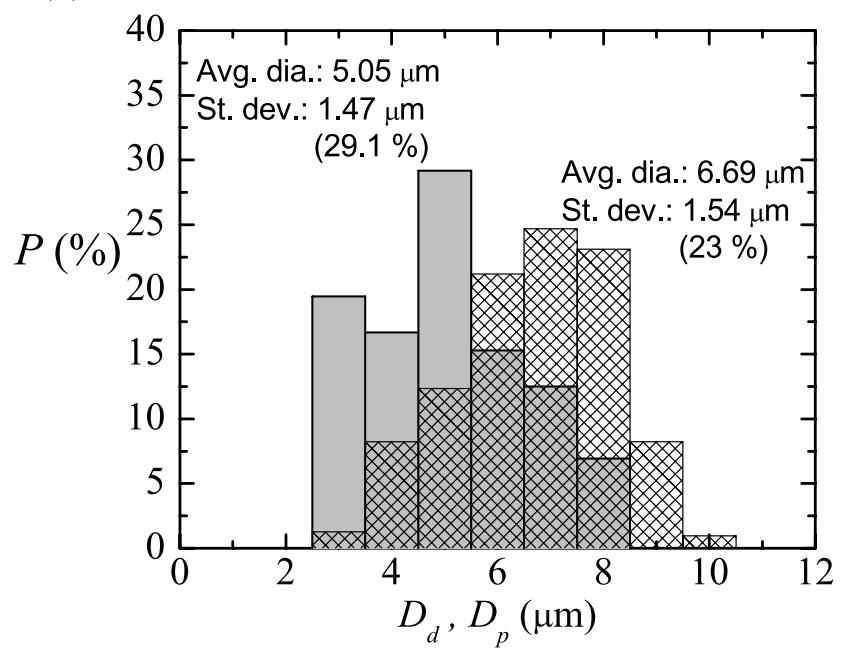

(c)

Figure 2. Probability distribution $\mathcal{P}\left(D_{d}\right)$ for the primary liquid droplet diameter $D_{d}$ (meshed bars) and $\mathcal{P}\left(D_{p}\right)$ for the solid particle diameter after curing $D_{p}$ (gray bars) for three different experimental realizations/samples (a)-(c). Ratio of the PDMS precursor, Part A:Part B, of 6:4 [7]. Reprinted from [7], with the permission of AIP Publishing.

Muñoz-Sánchez et al [7] found shrinkage to be important for PDMS spherical microparticles while curing. In that case, the unusually high amount of curing agent appeared as a possible candidate to induce the shrinkage of the samples.

To check the influence of the amount of curing agent on the results in [7], experiments were conducted to measure the size of liquid primary droplets and subsequent solid particles (just after curing) for several cases, using different proportions Part A:Part B of a common PDMS elastomer kit (Dow Corning Sylgard 186 Silicone Elastomer Kit) formed by a base of vinyl-terminated siloxane oligomers (Part A) and a curing agent of siloxane oligomers and catalyst (Part B). The ratios Part A:Part B that we analysed were: 6:4 (from [7]), 8:2, and 10:1, whose viscosities were around $827 \mathrm{cSt}, 1700 \mathrm{cSt}$, and $3196 \mathrm{cSt}$, respectively. Figures $2-4$ show the probability distributions $\mathcal{P}\left(D_{d}\right)$ for the primary liquid droplet diameter $D_{d}$ and $\mathcal{P}\left(D_{p}\right)$ for the solid particle diameter after curing $D_{p}$, for three samples with different size of the primary droplets. Each figure corresponds to a different ratio Part A:Part B (6:4, $8: 2$ and 10:1). In all cases, either before or after curing, the coefficient of variance for the diameters $C_{v}, C_{v}=\frac{\sigma}{\overline{D_{d}}} 100$, is around $25 \%$, showing an acceptable degree of monodispersity.

The curing shrinkage coefficient $C_{S}$ was calculated as $C_{s}=\frac{\bar{D}_{d}-\bar{D}_{p}}{D_{d}} 100$ for all the cases. Figure 5 depicts the values for the samples in figures 2-4. $C_{S}$ increased linearly with the average liquid droplet diameter $\bar{D}_{d}$, for liquid droplet diameters approximately comprised between 0 and 24 microns. The points in this range fit properly to the equation $C_{s} \simeq \frac{10}{3} \bar{D}_{d}$ for all the ratios: 6:4 [7], 8:2, and 10:1 (see dashed line in that figure). This means that the pronounced shrinkage found in this technique to produce microparticles does not depend on the amount of curing agent in the PDMS precursor. Rather surface effects, while the particles are curing, would motivate this pronounced shrinkage due to the relatively large droplet surface-to-volume ratio. For each ratio Part A:Part B, the cured PDMS particles of each sample will have a different density, but a natural question is whether the properties of the particles (flexibility, etc) depend on the proportion of curing agent. 


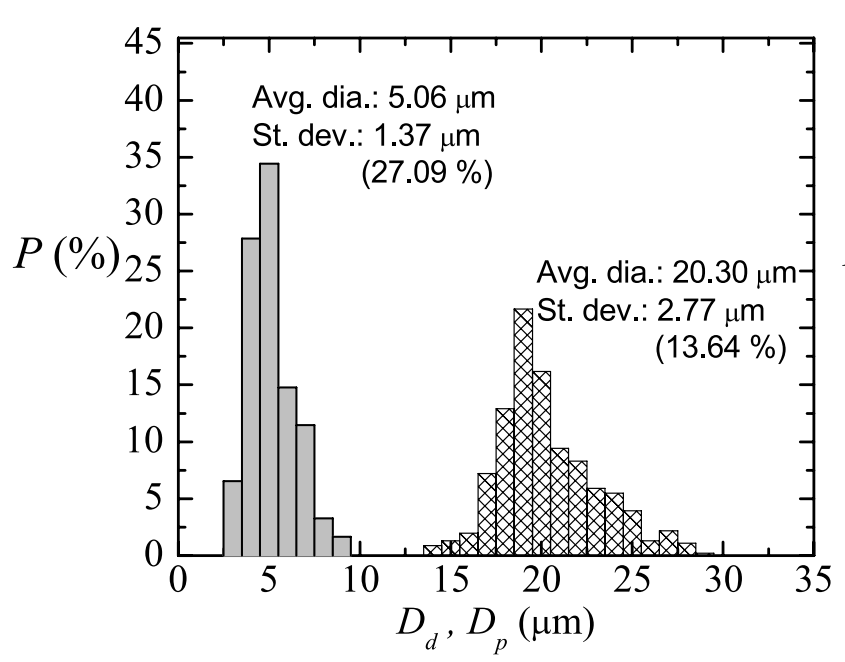

(a)

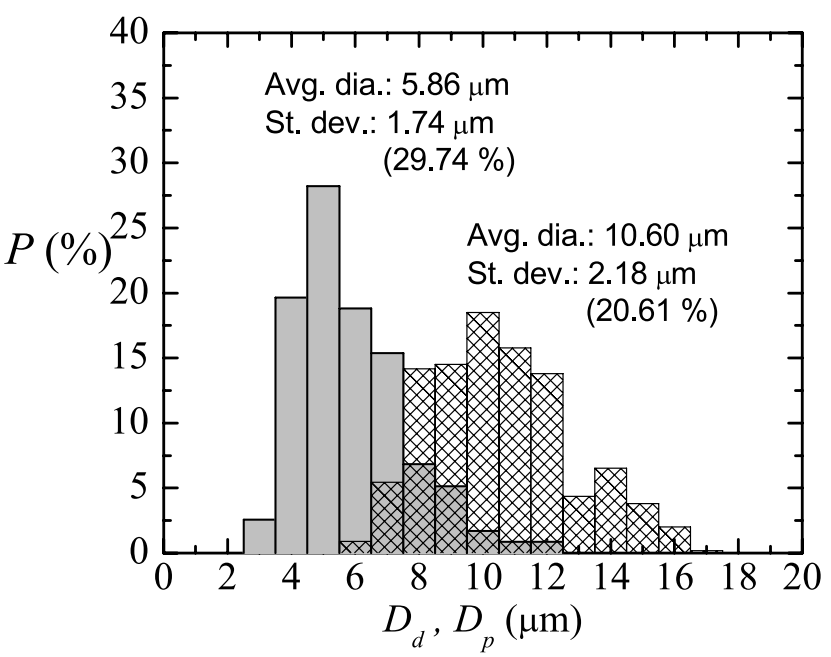

(b)

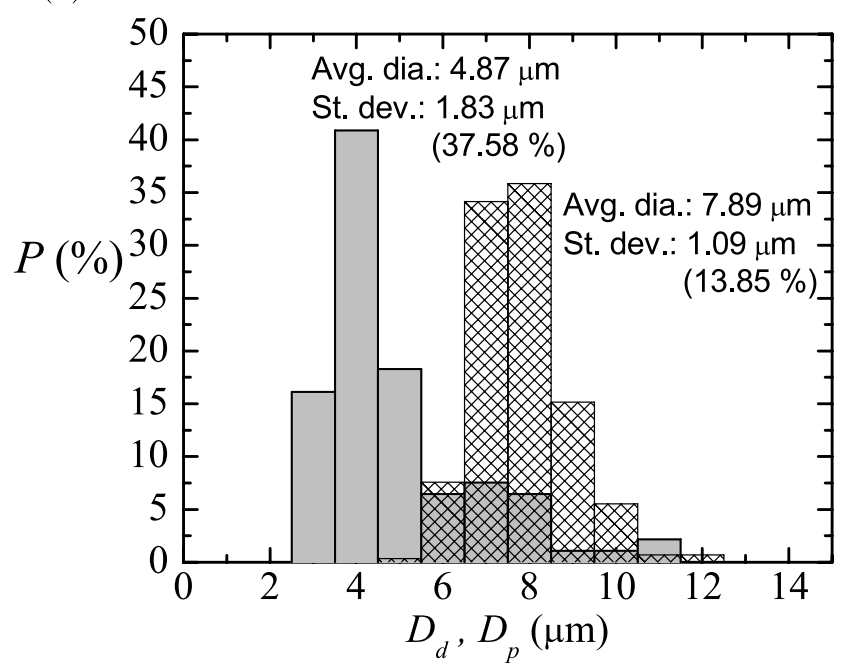

(c)

Figure 3. Probability distribution $\mathcal{P}\left(D_{d}\right)$ for the primary liquid droplet diameter $D_{d}$ (meshed bars) and $\mathcal{P}\left(D_{p}\right)$ for the solid particle diameter after curing $D_{p}$ (gray bars) for three different experimental realizations/samples (a)-(c). Ratio of the PDMS precursor, Part A:Part B, of 8:2.

To close this section and to complete the calibration/prediction of our shrinkage phenomenon, we developed experiments to obtain the shrinkage coefficient $C_{s}$ for higher droplet diameters, specifically, $\bar{D}_{d}=32.4$ and $56.2 \mu \mathrm{m}$. As one would expect, surface effects are negligible in these cases, and the shrinkage of the droplet is proportional to its volume, i.e. $D_{p} \simeq \alpha D_{d}(\alpha \simeq 0.2)$. Therefore, $C_{s}$ reaches a constant value around $80 \%$ for $\overline{D_{d}}$ above about $24 \mu \mathrm{m}$, as can be seen in figure 5 (dotted line). It should be pointed out that between these two behaviors (linear dependency and constant value of $C_{s}$ ) there should be a transition region. As an estimation, this region will take place for $\bar{D}_{d}$ around $24 \mu \mathrm{m}$, i.e. the intersection point of the two lines in figure 5.

\section{Colour}

Figure 1 shows the transparent microparticles obtained from the technique proposed in [7], by using the common PDMS precursor. The contours of the particles are clearly detected in these images, but for other applications/measurements it would be desirable to add colour to such small particles, thus enhancing contrast and making their visualization easier. Just a few works can be found in literature about colouring polydimethylsiloxane. Park et al [25] demonstrated that embedding silicon nanowires into PDMS presented a way to add colour, as they provided filtering at visible and nearinfrared wavelengths. Khane et al [26] used a commercial pigment for paints to fabricate colored PDMS micropillar arrays for high-throughput measurements of forces applied by genetic model organisms. To the best of the authors' knowledge, there is no specific colourant, i.e. a soluble dye, for PDMS, so the use of pigments seems the most reasonable solution. By definition, a pigment is not soluble in water or oil, so the expected outcome when mixing PDMS precursor with the pigment is a fine dispersion/emulsion.

First we performed preliminary colouring tests with eight different commercial dyes/pigments. The dye/pigment was carefully mixed with part A, and part B was then added to obtain at the end a PDMS precursor mixture. Figure 6 shows our dyed PDMS mixtures after curing. Although PDMS 


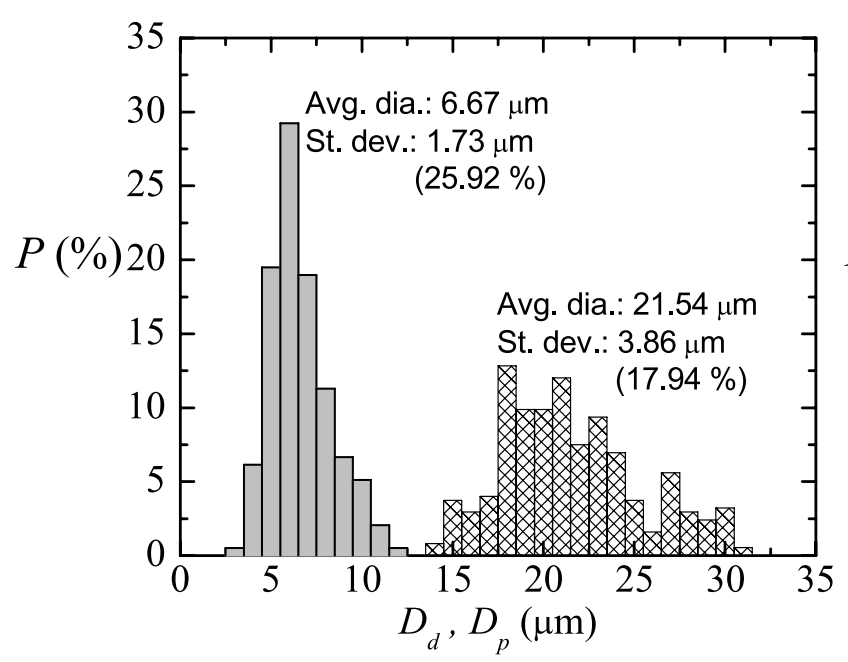

(a)

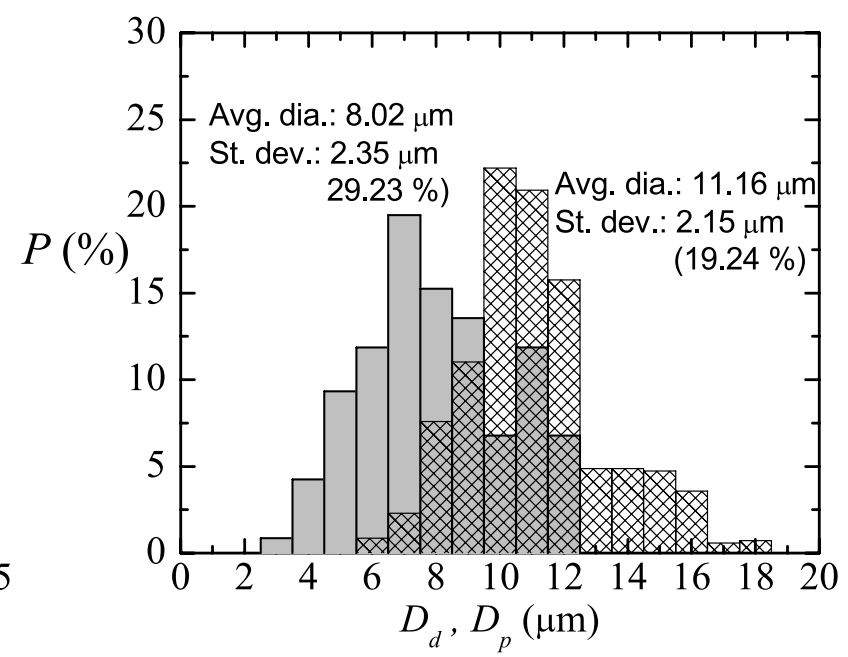

(b)

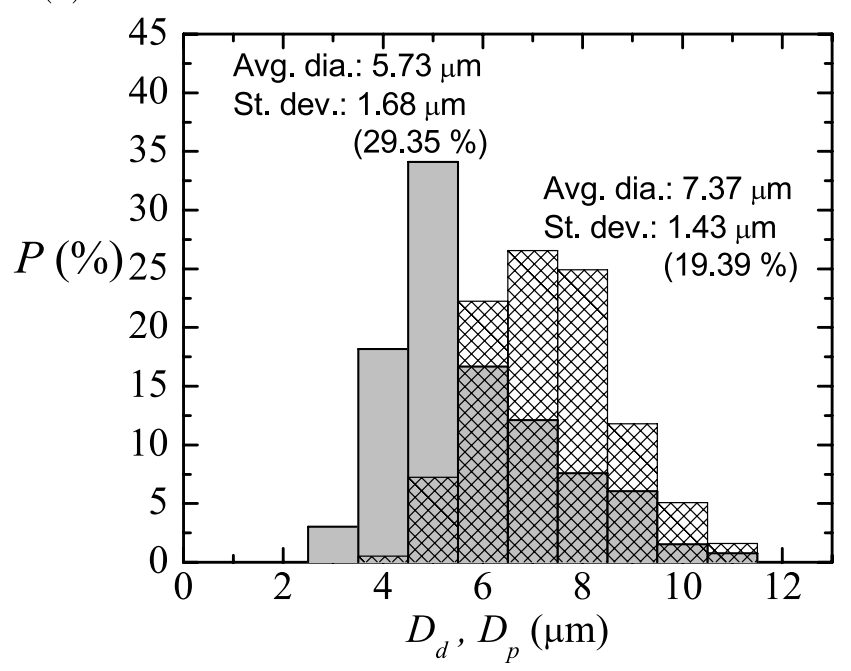

(c)

Figure 4. Probability distribution $\mathcal{P}\left(D_{d}\right)$ for the primary liquid droplet diameter $D_{d}$ (meshed bars) and $\mathcal{P}\left(D_{p}\right)$ for the solid particle diameter after curing $D_{p}$ (gray bars) for three different experimental realizations/samples (a)-(c). Ratio of the PDMS precursor, Part A:Part B, of $10: 1$

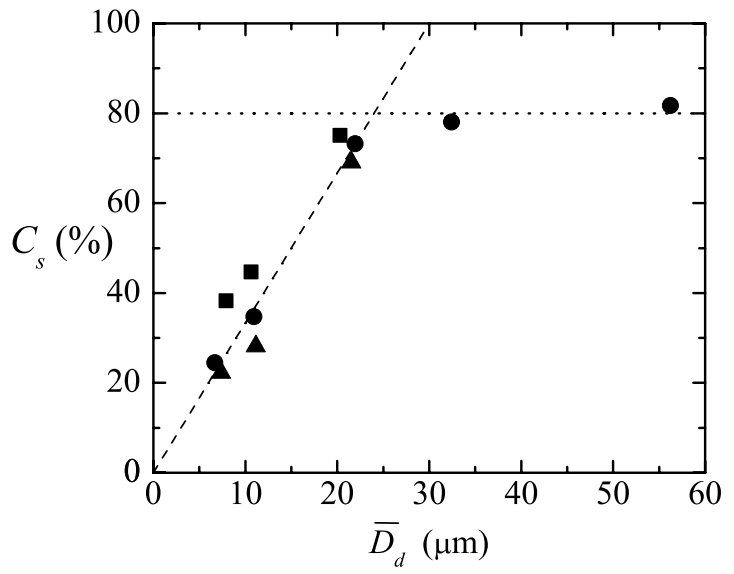

Figure 5. Curing shrinkage coefficient $C_{s}$ of the PDMS liquid droplets after curing as a function of the original diameter in liquid phase $\overline{D_{d}}$ for the ratios 6:4 (circles) [7], 8:2 (squares), and 10:1 (triangles). The dashed line indicates a possible fit line to predict this shrinkage, $C_{s} \simeq \frac{10}{3} \overline{D_{d}}$, when $\overline{D_{d}}$ ranges between 0 and $24 \mu \mathrm{m}$ approximately [7]. The dotted line marks the constant value reached in the shrinkage coefficient for bigger droplets. samples appear to be uniformly coloured when observed at a millimetric scale, at a microscale level the microstructure results revealed that most of these samples need further improvement. In the samples (a)-(d), spheres of food dye with diameters between 1-100 $\mu \mathrm{m}$ were found trapped in the solidified PDMS mass. Solid aggregations of particles with sizes larger than $10 \mu \mathrm{m}$ can be seen in sample (e) because this pink pigment is commercialized in powder format. The result of the blue pigment (f) is similar to the previous one. Only the last two samples (g) and (h) showed a more homogenous and suitable microstructure for the production of coloured micro-sized particles, so the PDMS precursor dyed with these pigments was selected to generate the microparticles.

Our technique was used for the production of microdrops after introducing these two pigments in the PDMS precursor (10:1), where different amounts of the dye were also tested. In figure 7 it can be seen the droplets of coloured PDMS for several cases in the moment of their production. In case (a) and (b) it was added $3 \mathrm{wt} \%$ of white pigment (paste of FINE WHITE 

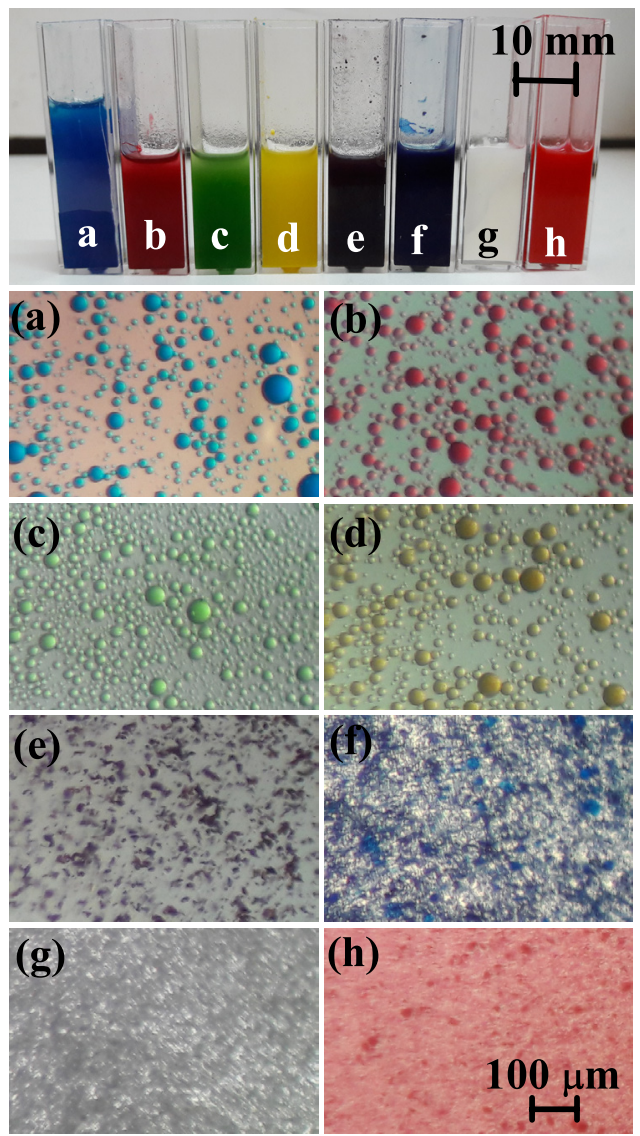

Figure 6. (On the top) samples of cured coloured PDMS in plastic cuvettes. The amount of dye was around $4 \mathrm{wt} \%$ for all the cases: (a) blue, (b) red, (c) green, and (d) yellow food dye (Dr Oetker colour gels), (e) fluorescent pink pigment (water-soluble powder of ROSA BTE. HISPACID B-250\%, Robama), (f) blue pigment (paste of AZUL HISPALIT HL-HB PASTA-170\%, Robama), white pigment (paste of FINE WHITE 7006, Eastern Chemicals), and red pigment for silicone (paste of PP SIL RO-1, Plastiform). The zoomed images below show the resulting microstructure for each mixture, obtained by standard microscopy of a cured drop on a glass slide.

7006, Eastern Chemicals) and red pigment for silicone (paste of PP SIL RO-1, Plastiform), respectively. Comparing both results, it is clear that the microentities of pigment trapped in the drops of case (a) are too large for our purpose, reaching sizes of even a few microns, which results in a heterogeneous colouration of the droplet. However, in case (b) the coloration is more homogeneous because the nanoentities of pigment are well distributed in the bulk of the droplet. For this reason, it was decided to rule out the white pigmet for the production of PDMS microparticles. However, it should be noted that the amount of pigment was insufficient to generate doplets with strong colour in both cases. In case (c), it was possible to produce distintive coloured droplets of PDMS by increaing the amount of red pigment (similar amount used in [26]). In other words, the more amount of pigment increases the particles of pigment inside the PDMS droplets. We analysed the probability distributions $\mathcal{P}\left(D_{d}\right)$ for the primary liquid droplet diameter $D_{d}$ and $\mathcal{P}\left(D_{p}\right)$ for the solid particle diameter after curing $D_{p}$ for one sample of this last coloured PDMS precursor, with viscosity of around $1400 \mathrm{cSt}$, see figure 8 . In

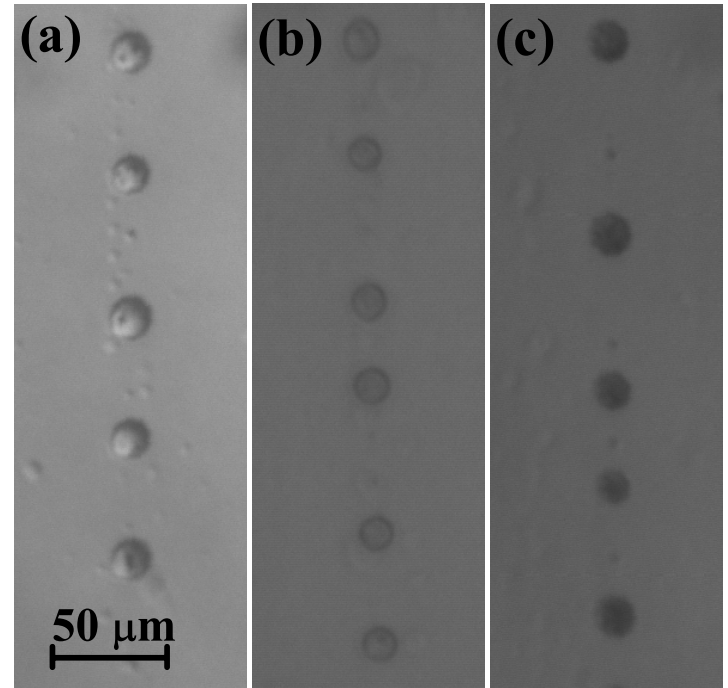

Figure 7. Droplets of PDMS (10:1) pigment-dyed precursor travelling through the glass capillary in the moment of their production: (a) $3 \mathrm{wt} \%$ of white pigment (paste of FINE WHITE 7006, Eastern Chemicals), (b) 3 wt\% of red pigment for silicone (paste of PP SIL RO-1, Plastiform) and (c) $20 \mathrm{wt} \%$ of the last one. All the droplets in the images have a diameter around $15 \mu \mathrm{m}$.

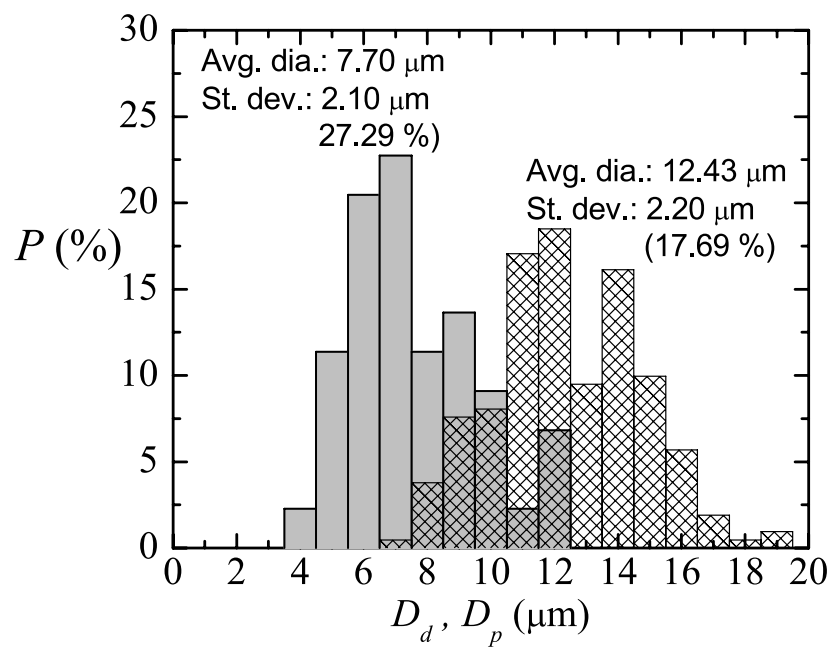

Figure 8. Probability distribution $\mathcal{P}\left(D_{d}\right)$ for the primary liquid droplet diameter $D_{d}$ (meshed bars) and $\mathcal{P}\left(D_{p}\right)$ for the solid particle diameter after curing $D_{p}$ (gray bars). Ratio 10:1 with $20 \mathrm{wt} \%$ of red pigment for silicone (paste of PP SIL RO-1, Plastiform).

spite of the addition of $20 \mathrm{wt} \%$ of red pigment, the results are similar to those shown in figure 4 without any pigment.

On the other hand, we also tested a commercial coloured PDMS, Sylgard 170 Silicone Elastomer (Dow Corning) and called 'black' PDMS in the text (with viscosity when mixed of around $2200 \mathrm{cSt}$ ), to compare the results to those of our 'homemade' coloured PDMS. Figure 9 shows the production of droplets with this black PDMS. The emitted jet of PDMS precursor attached to the needle tip revealed that this material is a dispersion as expected, i.e. it is made of PDMS with a special pigment. In fact, the physical appearance of the droplets resulting are similar to those in figure 7(c). In figure 10, one can be found the results of the probability distributions $\mathcal{P}\left(D_{d}\right)$ for the primary liquid droplet diameter $D_{d}$ and $\mathcal{P}\left(D_{p}\right)$ 

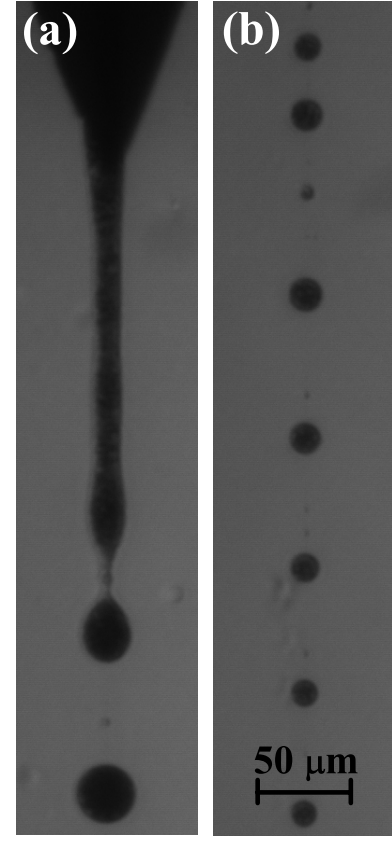

Figure 9. Production of droplets with Sylgard 170 Silicone Elastomer (Dow Corning), ‘black' PDMS, (1:1). The emitted jet of PDMS precursor attached to the needle tip (a) and droplets of diameter around $15 \mu \mathrm{m}(\mathrm{b})$.

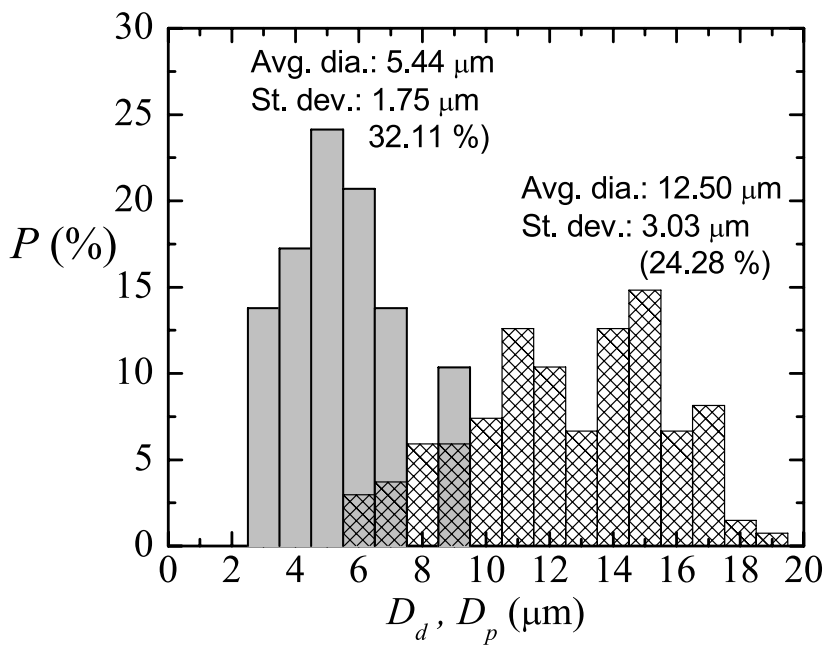

Figure 10. Probability distribution $\mathcal{P}\left(D_{d}\right)$ for the primary liquid droplet diameter $D_{d}$ (meshed bars) and $\mathcal{P}\left(D_{p}\right)$ for the solid particle diameter after curing $D_{p}$ (gray bars). Ratio 1:1 of Sylgard 170 Silicone Elastomer (Dow Corning), 'black' PDMS.

for the solid particle diameter after curing $D_{p}$ for one sample of this black PDMS. Again, the results are quite similar to those shown in figure 4 without any pigment.

It was calculated the curing shrinkage coefficient $C_{s}$ for several samples of coloured PDMS, as can be seen in figure 11. The results show that the addition of a pigment to the PDMS mixture, i.e. the addition of micro/nanoparticles, does not seem to change the rules of shrinkage of the particles produced by using our technique, at least for the amounts used in this work. The new points are in a good agreement with the lines of figure 5 in section 2 . However, it should be noted that this addition of pigment will have influence on the properties

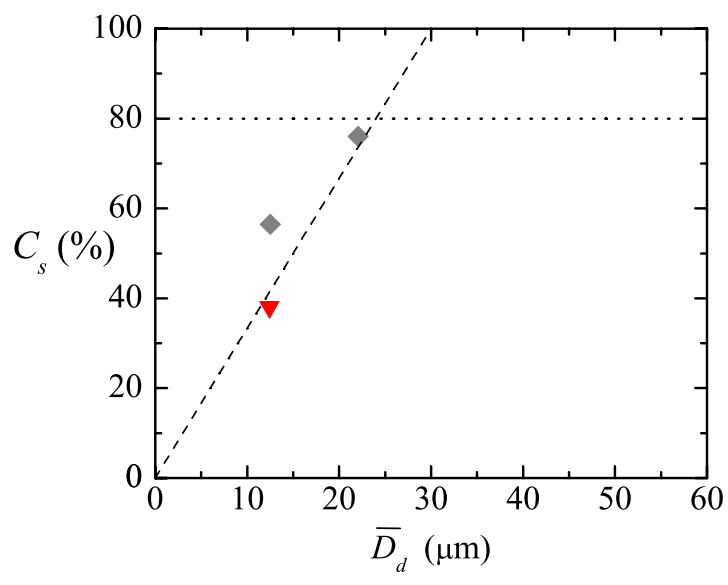

Figure 11. Curing shrinkage coefficient $C_{S}$ of the PDMS liquid droplets after curing as a function of the original diameter in liquid phase $\bar{D}_{d}$ for coloured PDMS: ratio 10:1 with $20 \mathrm{wt} \%$ of red pigment for silicone (paste of PP SIL RO-1, Plastiform) (red downtriangle) and ratio 1:1 of Sylgard 170 Silicone Elastomer (Dow Corning), ‘black' PDMS (grey diamond). Lines from figure 5.

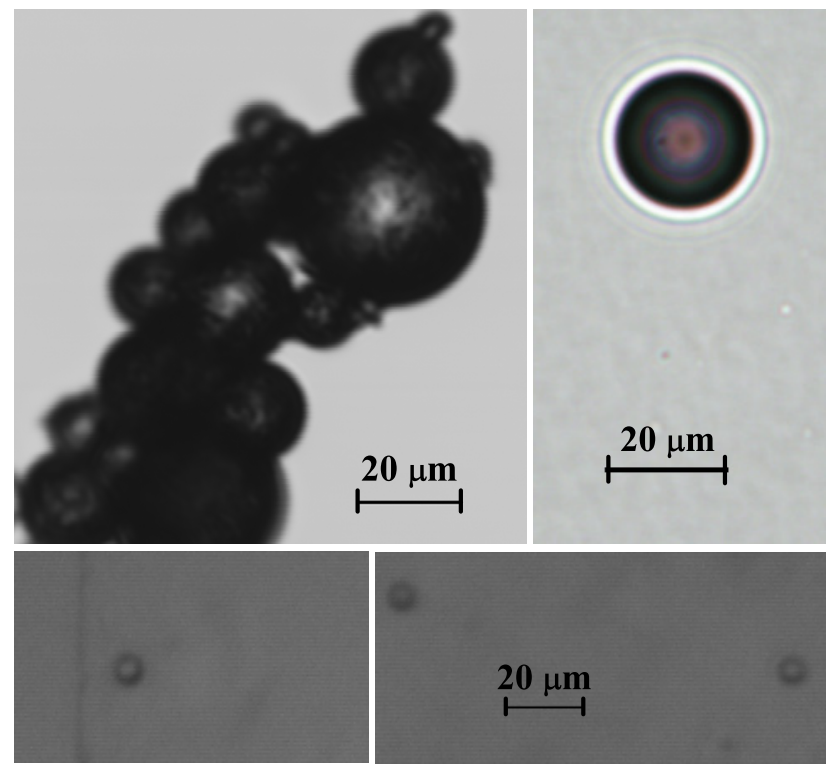

Figure 12. Coloured cured particles: (top-left) image of particles of Sylgard 170 Silicone Elastomer (Dow Corning), 'black' PDMS, taken by using a confocal microscope (FV1000 Olympus), (topright) image of particles of standard PDMS with $20 \mathrm{wt} \%$ of red pigment for silicone (paste of PP SIL RO-1, Plastiform) taken by using a direct microscope (BX51 Olympus). The images in the bottom show the same type of 'black' (left) and 'red' (right) particles visualized by using our backlight optical imaging.

of the PDMS after curing. Khane et al [26] observed a large reduction in the stiffness of PDMS on addition of dye. Taking into account these results, one might use our calibrated technique to microencapsulate, in a relatively controlled way, other type of desirable micro/nanoparticles within the PDMS droplets, for example, nanoparticles with electric, magnetic or fluorescent properties. Thus, our technique could be used to produce microcapsules of a few microns in diameter for microfluidic applications ranging from microcooling to magnetic hyperthermia. 
Finally, it is worth mentioning that by using our backlight imaging, the coloured particles (after curing) looked clear, almost without colour, just the visualization of their contours (free surface) was enhanced. The real colour of our particles was checked by using other microscopes, see figure 12 . The extremely small size of our coloured solid particles and the change in the diffraction of the light when coloured droplets get cured might cause the 'loss' of colour when standard backlight optical imaging is used.

\section{Conclusions}

In this work, we have calibrated the shrinkage phenomenon taking place in the production of PDMS microparticles, when using the technique proposed by Muñoz-Sánchez et al [7]. The shrinkage of our samples did not depend on the amount of curing agent in the PDMS precursor and, in principle, on the addition of micro/nanoparticles to the mixture.

Besides, we have developed a protocol to fabricate a low-cost 'homemade' coloured PDMS, improving the visualization/detection of the particles. The addition of colour by using pigments has enhanced the visualization of the contours of the PDMS microparticles, and has revealed the capability of this technique to microencapsulate micro/nanoparticles in PDMS spheres of a few microns in diameter. These microparticles can be used in a wide range of technological fields, such as biomedicine, biotechnology, pharmacy, and industrial engineering.

Finally, it must be pointed out that this technique was able to work with a wide range of viscosities (from less than 800 to around $4000 \mathrm{cSt}$ ), obtaining in all the cases micro-size particles with a relatively high degree of monodispersity.

\section{Acknowledgment}

The authors acknowledge the financial support provided by Fundação para a Ciência e a Tecnologia (FCT), COMPETE2020 through projects UID/EMS/00532/2013, UID/EMS/04077/2013, POCI-01-0145-FEDER-016861 (with associated reference PTDC/QEQ-FTT/4287/2014) and fellowships SFRH/BD/89077/2012 and SFRH/BSAB/135419/2017. Partial support from the spanish Ministry of Science and Education (grant no. DPI2016-78887), Gobierno de Extremadura (grant no. GR10047), and "la Caixa" Foundation (predoctoral grant) is gratefully acknowledged too.

\section{ORCID iDs}

E J Vega (i) https://orcid.org/0000-0003-1891-0531

\section{References}

[1] Pinho D, Campo-Deaño L, Lima R and Pinho F T 2017 In vitro particulate analogue fluids for experimental studies of rheological and hemorheological behavior of glucose-rich rbc suspensions Biomicrofluidics 11054105
[2] Calejo J, Pinho D, Galindo-Rosales F J, Lima R and Campo-Deaño L 2016 Particulate blood analogues reproducing the erythrocytes cell free layer in a microfluidic device containing a hyperbolic contraction Micromachines 74

[3] Bento D, Sousa L, Yaginuma T, Garcia V, Lima R and Miranda J M 2017 Microbubble moving in blood flow in microchannels: effect on the cell-free layer and cell local concentration Biomed. Microdevices 196

[4] Rodrigues O R, Bañobre-López M, Gallo J, Tavares P B, Silva A M T, Lima R and Gomes H T 2016 Haemocompatibility of iron oxide nanoparticles synthesized for theranostic applications: a high-sensitivity microfluidic tool J. Nanopart. Res. 18194

[5] Faustino V, Catarino S O, Lima R and Minas G 2016 Biomedical microfluidic devices by using low-cost fabrication techniques: a review J. Biomech. 49 2280-92

[6] Rodrigues R O, Pinho D, Bento D, Lima R and Ribeiro J 2016 Wall expansion assessment of an intracranial aneurysm model by a $3 \mathrm{~d}$ digital image correlation system Measurement 88 262-70

[7] Muñoz-Sánchez B N, Silva S F, Pinho D, Vega E J and Lima R 2016 Generation of micro-sized PDMS particles by a flow focusing technique for biomicrofluidics applications Biomicrofluidics 10014122

[8] Gañán-Calvo A M, Montanero J M, Martín-Banderas L and Flores-Mosquera M 2013 Building functional materials for health care and pharmacy from microfluidic principles and flow focusing Adv. Drug Deliv. Rev. 65 1447-69

[9] Zhao L-B et al 2011 A novel method for generation of amphiphilic PDMS particles by selective modification Microfluid Nanofluid 10 453-8

[10] Jiang K, Thomas P C, Forry S P, DeVoe D L and Raghavan S R 2012 Microfluidic synthesis of monodisperse pdms microbeads as discrete oxygen sensors Soft Matter 8 923-6

[11] Carneiro J, Doutel E, Campos J B L M and Miranda J M 2016 PDMS droplet formation and characterization by hydrodynamic flow focusing technique in a pdms square microchannel J. Micromech. Microeng. 26105013

[12] do Nascimento D F, Avendaño J A, Mehla A, Moura M J B, Carvalho M S and Duncanson W J 2017 Flow of tunable elastic microcapsules through constrictions Sci. Rep. 711898

[13] Acero A J, Rebollo-Muñoz N, Montanero J M, GañánCalvo A M and Vega E J 2013 A new flow focusing technique to produce very thin jets J. Micromech. Microeng. 23065009

[14] Sousa P C, Pinho F T, Oliveira M S N and Alves M A 2011 Extensional flow of blood analog solutions in microfluidic devices Biomicrofluidics 5014108

[15] Campo-Deaño L, Dullens R P A, Aarts D G A L, Pinho F T and Oliveira M S N 2013 Viscoelasticity of blood and viscoelastic blood analogues for use in polydymethylsiloxane in vitro models of the circulatory system Biomicrofluidics 7034102

[16] Kalambur V S, Han B, Hammer B E, Shield T W and Bischof J C 2005 In vitro characterization of movement, heating and visualization of magnetic nanoparticles for biomedical applications Nanotechnology 16 1221-33

[17] Saidur R, Leong K Y and Mohammad H A 2011 A review on applications and challenges of nanofluids Renew. Sust. Energ. Rev. 15 1646-68

[18] Yi P, Khoshmanesh K, Campbell J L, Coughlan P, Ghorbani K and Kalantar-Zadeh K 2014 Investigation of different nanoparticles for magnetophoretically enabled nanofin heat sinks in microfluidics Lab Chip 14 1604-13

[19] Khullar V, Tyagi V H, Hordy N, Otanicar T P, Hewakuruppu Y, Modi P and Taylor R A 2014 Harvesting 
solar thermal energy through nanofluid-based volumetric absorption systems Int. J. Heat Mass Transf. 77 377-84

[20] Verma S K and Tiwari A K 2015 Progress of nanofluid application in solar collectors: a review Energy Conv. Manag. 100 324-46

[21] Moraes C, Sun Y and Simmons C A 2009 Solving the shrinkageinduced PDMS alignment registration issue in multilayer soft lithography J. Micromech. Microeng. 19065015

[22] Lee S W and Lee S S 2007 Shrinkage ratio of PDMS and its alignment method for the wafer level process Microsyst. Technol. 14 205-8

[23] Jeong O C and Konishi S 2011 Controlling the size of replicable polydimethylsiloxane (PDMS) molds/stamps using a stepwise thermal shrinkage process Microelectron. Eng. $882286-9$

[24] Lee J N, Park C and Whitesides G M 2003 Solvent compatibility of poly(dimethylsiloxane)-based microfluidic devices Anal. Chem. 75 6544-54

[25] Park H, Seo K and Crozier K B 2012 Adding colors to polydimethylsiloxane by embedding vertical silicon nanowires Appl. Phys. Lett. 101193107

[26] Khare S M, Awasthi A, Venkataraman V and Koushika S P 2015 Colored polydimethylsiloxane micropillar arrays for high throughput measurements of forces applied by genetic model organisms Biomicrofluidics 9014111 